may be kept accurately in focus during the period required to complete the observations. The main features of the apparatus being shown by the illustration, a brief description will suffice. The parabolic reflector which concentrates the rays from the chromosphere (described in the previous article) is placed in the cavity of a conical dish of cast-iron, secured to the top of a table suspended on two horizontal journals, and revolving on a vertical axle. The latter, slightly taper, turns in a cast-iron socket which is bushed with brass and supported by three legs stepped on a triangular base, resting on friction-rollers. The horizontal journals referred to turn in bearings attached to a rigid bar of wrousht-iron situated under the table, firmly secured to the upper end of the vertical axle. The horizontal angular position of the table is adjusted by a screw operated by the small hand-wheel $a$, the inclination being regulated by another screw turned by the hand-wh $=\mathrm{el} b$. A graduated quadrant, $e$, is attached to the end of the table in order to afford means of ascertaining the sun's zenith distance at any moment. The index $d$, which marks the degree of inclination, is stationary, being secured to the rigid bar before described. The rays from the photosphere are shut out by a circular disc, $f$, composed of sheet metal turned to exact size, and supported by three diagonal rods of steel. These rods are secured to the circumference of the conical dish by screws and adjustable nuts in such a manner that the centre of the disc $f$ may readily be brought in a direct line with the axis of the reflector. The michanism adopted for adjusting the position of the table by the hand-wheels $a$ and $b$ requires no explanation; but the device which enables the operator to ascertain when the axis of the reflector is pointed exactly towards the centre of the sun demands particular notice. A shallow cylindrical box, $g$, provided with a flat lid and open at the bottom, excepting a narrow flange extending round the circumference, is firmly held by two columns secured to the top of the table. A convex lens of 26 inches focus is inserted in the cylindrical $b: x$, the narrow fiange mentioned affording necessary support. The lid is perforated by two openings at right angles, 0.05 inch wide, 2.5 inches long, forming a cross, the lens being so adjusted that its axis passes through the central point of intersection of the cross. The face of the table being turned at right angles to the sun, or nearly so, it will be evident that the rays passing through the perforations and through the lens will produce, at a certain distance, a brilliantly illuminated cross of small size and sharp outline. A piece of ivory, or white paper, on which parallel lines are drawn intersecting each other at right angles, is attached to the top of the table in such a position that the centre of intersection of the said lines coincides with the axis of the lens. This axis being parallel with the line passing through the centre of the disc $f$ and the focus of the reflector, it will be perceived that the operator, in directing the table, has only to bring the illuminated cross within the intersecting parallel lines on the piece of ivory. Ample practice has shown that by this arrangement an attentive person can easily keep the disc $f$ accurately in line with the focus of the refector and the centre of the sun during any desirable length of time. The absence of any perceptible motion of the column of the focal thermometer during the experiments which have been made furnishes the best evidence that the sun's rays have been effectually shut out by the intervening disc, which, it should be remembered, is only large enough to screen the aperture of the reflector from the rays projected by the photosphere. It may be noticed that actinometric observations cannot be accurately made unless the instrument is attached to a table capable of being directed in the manner described; nor is it possible to measure the dynamic energy transmitted by solar radiation unless the calorimeter employed for the purpose faces the sun with the same precision as our parabolic reflector. It is worthy of notice that the lightness of the illustrated apparatus ren- ders exact adjustment easy, since screws of small diameter and fine pitch may be employed. It only remains to be stated that in order to admit of accurate examination of the spectrum before referred to, the thermometer is removed during investigations which do not relate to temperature, a cylindrical stem of metal, 0.25 inch diameter, coated with lamp-black, being introduced in its place.

With reference to the result of recent experiments, it is proper to state that, at the present time, the sun's zenith distance being now nearly $60^{\circ}$ at noon, no perceptible heating takes place in the focus of the parabolic reflector. The observations relating to temperature mentioned in the previous article, were made when the zenith distance was only one-third of what it is at present. The consequent increase of atmospheric depth, at this time, has completely changed the colour of the spectrum, and rendered the same so feeble that its extent cannot be determined. As seen last summer, before the earth had receded far from the aphelion, the termination of the spectrum reached so far down that an addition of O'I 5 inch to the radius of the disc $f$ would scarcely have shut it out. Now an addition of $0^{\circ} 15$ inch to the radius of the disc corresponds to an angular distance of $9^{\prime} 45^{\prime \prime}$; hence, assuming the radius of the photosphere to be 426.300 miles, the depth of the solar atmosphere cannot be less than 255,000 miles. And, judging from the appearance at the period referred to, there can be little doubt that a larger and more perfect reflector will enable us to trace the spectrum still further down. Consequently, a further enlargement of the disc $f$ will be required to extinguish wholly the reflected light from the solar atmosphere. It is reasonable, therefore, to suppose that the depth of the solar atmosphere will ultimately be found to exceed very considerably the foregoing computation.

It has been suggested regarding the instituted investigations of the radiant heat transmitted by the chromosphere, that the thermo-electric pile ought to be employed in combination with the parabolic reflector. The object of the investigation being simply that of proving by the feebleness of the radiant power transmitted to the surface of the earth that the chromosphere and outer strata of the sun's envelope do not possess radiant energy of sufficient intensity to influence solar temperature as supposed by Secchi, tests of the suggested extreme nicety are not called for

With reference to the effect of increased depth, the small amount of retardation suffered by the rays in passing through the highly attenuated atmosphere of the sun, previously established, shows that the question of solar temperature will not be materially affected, even should it be found that the depth of the envelope is greater than the radius of the photosphere.

\section{J. ERICSSON}

\section{THE RIGIDITY OF THE EARTH}

SIR WILLIAM THOMSON'S views regarding the rigidity of the earth have been hitherto received in silence by those who entertain different opinions from him; but it does not follow on this account that they regard his position as unassailable. It is more satisfactory to attempt to establish positive results in science, than to criticise the labours of others; but as Sir William Thomson, by his letter in NATURE for January I8, manifestly invites discussion, I hope I shall be excused for making the following remarks.

When nearly ten years since I saw the abstract in the Proceedings of the Royal Society which he appends to his letter, I resolved to suspend my judgment until I had an opportunity for reading his papers in extenso. To such of your readers as happen to be interested in this question, and who have not yet seen these publications, I would venture to recommend a similar course, In the 
"Philosophical Transactions" for 1862 , the memoir on the rigidity of the earth is fully printed, and immediately following it is another designated "dynamical problems regarding elastic spheroidal shells and spheroids of incompressible liquid." The conclusions arrived at in the first are essentially and admittedly dependent on the investigations presented in the second. Not long after they were published I gave my best attention to the study of both, and it soon appeared to me that the problems treated in the second could have no physical bearing on the question of the earth's structure. The very title of this memoir partly reveals its character in this respect. In order to apply the results obtained in this memoir to the earth, it is supposed to be a spheroidal homogeneous elastic shell filled with incompressible fluid; whereas in such an inquiry the earth can scarcely be supposed to be otherwise than a heterogeneous solid envelope containing a fluid whose properties are not inconsistent with those of fluids coming under our notice. Under this form I have treated the hypothesis in the "Philosophical Transactions" for $185 \mathrm{r}$, and also in subsequent publications.

Incompressibility is not a property of any known fluid; and Neumann, when referring in his comprehensive treatise on geology to the influence of pressure in promoting the density of the interior parts of the earth, expresses what is very generally admitted among philosophical geologists as well as physical inquirers, when he says that "fluid bodies are endowed with far more compressibility than solids." * Hypotheses are often indispensable in physical inquiries where we are proceeding from the known to the unknown, but there are two conditions to which they should conform; first, they should be capable of verification by a comparison of the results to which they lead with those of observation, and secondly, they should not contradict established physical laws or the known properties of matter, unless the contradiction is specially explained and fully accounted for. The second of these conditions is clearly violated when the internal fluid of the earth is supposed to differ from all known fluids by being supposed to be incompressible. And this violation is especially flagrant when the solid matter enclosing the incompressible fluid is supposed to be at the same time elastic and therefore compressible, and when, moreover, the line of reasoning adopted as to the earth's internal structure pointedly depends upon these assumptions as to the properties of its fluid and solid portions. Sir William Thomson endeavours to prove, by a process of reductio ad absurdum that the interior of the earth is for the most part or altogether solid ; in other words, he supposes the interior to be fluid, and then tries to show that the tidal actions produced in this fluid by the sun and moon must cause oscillations in the crust which have not been observed. He may justly clain to have proved that the earth does not consist of an elastic solid envelope enclosing a mass of the ideal substance called an incompressible liquid, but he has not proved the point which he intended to establish, namely, the absence of an interior fluid nucleus endowed with the properties commonly attributed to fluids. He also supposes throughout his investigations, in the same manner as was supposed by Mr. Hopkins, that the transition from the solidity of the shell to the fluidity of the nucleus is not gradual but abrupt. Those who maintain the validity of the hypothesis of the interior fluidity of the earth are far from holding this opinion. On the contrary, all observations hitherto made on the materials of the earth lead to the conclusion that the solid shell is so constituted as to present first a superficial coating whose mechanical properties we can partly ascertain by direct experiment; secondly, a mass whose density and rigidity probably increase with the depth from the outer surface; thirdly, an interior coating in which the effects of pressure are resisted by those of temperature, and where an imperfectly

* Lehrbuch der Geologie, i. p. 268, and edition. fluid and pasty mass is in contact at one side with the solid shell, and on the other with the more perfect fluid. This mass should be manifestly much more yielding and compressible than the perfectly solidified shell; for if compression tends to increase the rigidity of solid matter, the middle division of the shell, as just described, should be more rigid than its superficial portion, and very much more rigid than the interior pasty mass. The work performed by small changes of shape in the fluid nucleus due to the action of exterior disturbing bodies should thus be expended partly in producing small variations of density among the compressible strata of which it is composed, and partly in changing the shape of the yielding matter of the inner surface of the shell. The deformations of a shell consisting of homogeneous elastic matter, such as steel acted upon by exterior forces, must be the resultants of all the elementary deformations among its particles summed up or integrated. It would behave somewhat like a vibrating bell; but such is not the behaviour to be expected in a mass of discontinuous and heterogeneous materials. Vibratory motions in such bodies are for the most part extinguished by interferences, or their amplitudes are at least very much reduced.

If the conclusions deduced by M. Perrey of Dijon from his voluminous labours so often referred to by Mr. Mallet in his Reports on Earthquakes, be correct, some connection between these disturbances and the phases of the moon seems to be established which may be due to such comparatively feeble vibratory actions. Sir William Thomson's conclusions rightly interpreted show that the constitution of the fluid nucleus and the nature of the materials of the shell must be essentially different from what he supposes in order to establish these conclusions. A person who never saw a railway train might as justly reason as to the impossibility of travelling in it at high rates of speed, by demonstrating that the shocks experienced by perfectly rigid carriages connected without any compressible arrangements would be too great for travellers to endure, if not too great for the permanent integrity of the carriages themselves. In assuming the incompressibility of the fluid nucleus for the purposes of his indirect demonstration of the rigidity of the earth, Sir William Thomson makes a petitio principii nearly as vital as shocks incident to influence of buffers in reasoning on the the omission of the railway carriages.

I am at a loss to know where any warrant was found for affixing the property of incompressibility to the supposed fluid nucleus of the earth ; and those who maintain the hypothesis of the interior fluidity of the earth are entitled to repudiate an assumption fastened on that hypothesis not only in opposition to evidence derived from experiments on fluids, but in direct contradiction to the arguments employed by them in discussing the question of the earth's structure.

\section{HENRY HENNESSY}

\section{THE LANDSLIPS AT NORTHWICH}

IN the "Notes" of the number of NATURE, for Jan. 25, I find one referring to the landslips at Northwich in Cheshire, by mistake called Nantwich. As the description given of these landslips and their cause is scarcely accurate, your readers may like to see a short account of them.

Northwich is the great centre of the Cheshire salt trade. The manufacture is principally carried on now at Northwich and Winsford, both towns lying in the valley of the River Weaver, though formerly Nantwich was engaged in this trade, and Middlewich still continues so to be. The position of the latter is indicated by its name, it lying between Northwich and Nantwich. The salt is found lying in two beds, called the upper and lower rock salt. The first bed is met with in the neighbourhood of Northwich at the depth of about forty yards, and 